PROCEEDINGS OF THE

AMERICAN MATHEMATICAL SOCIETY

Volume 130, Number 3, Pages 661-665

S 0002-9939(01)06113-5

Article electronically published on August 29, 2001

\title{
UNMIXED LOCAL RINGS WITH MINIMAL HILBERT-KUNZ MULTIPLICITY ARE REGULAR
}

\author{
CRAIG HUNEKE AND YONGWEI YAO
}

(Communicated by Wolmer V. Vasconcelos)

Abstract. We give a new and simple proof that unmixed local rings having Hilbert-Kunz multiplicity equal to 1 must be regular.

\section{INTRODUCTION}

In 1969, Kunz Ku1 introduced a new numerical function of a Noetherian local ring in positive prime characteristic. One of Kunz's main results was the following characterization of regular local rings in positive prime characteristic $p$. We always let $q$ denote a varying power of $p$, and for an ideal $I$ write $I^{[q]}=\left(i^{q} \mid i \in I\right) R$.

Theorem 1.1 (Ku1 Proposition 3.2, Theorem 3.3]). Let $(R, \mathbf{m})$ be a Noetherian local ring of positive characteristic $p>0$. Then

(1) $\lambda_{R}\left(R / \mathbf{m}^{[q]}\right) \geq q^{d}$ for all $q=p^{e}(e \geq 1)$.

(2) $R$ is regular iff for some $q=p^{e}$ with $e \geq 1$ (equivalently for all $q$ ), $\lambda_{R}\left(R / \mathbf{m}^{[q]}\right.$ ) $=q^{d}$.

In [Ku2] Kunz observed that the function $q: \rightarrow \lambda_{R}\left(R / \mathbf{m}^{[q]}\right) / q^{d}$ should give interesting information about the nature of the singularity of $R$. His interest was at least partly inspired by searching for numerical invariants of singularities which might behave well under blowing up, as a means to understand resolution of singularities in positive characteristic. The idea of taking the limit of these numbers as $q$ goes to infinity was discussed by Kunz, and that the limit exists was shown by Monsky. He introduced the following definition:

Definition $1.2(\underline{\mathrm{M}}])$. Let $(R, \mathbf{m})$ be a Noetherian local ring of dimension $d$ and positive prime characteristic $p$. Let $M$ be a finitely generated $R$-module, and $I$ an m-primary ideal. Define

$$
e_{H K}(I, M)=\underset{q}{\lim _{q}} \frac{\lambda\left(M / I^{[q]} M\right)}{q^{d}} .
$$

If the dimension of $M$ is strictly less than the dimension of $R$, this limit is 0 . When $\operatorname{dim}(M)=\operatorname{dim}(R)$, the limit always exists and is a positive real number (Monsky [Mo]). By definition the Hilbert-Kunz multiplicity of $R$ is $e_{H K}(R)=$

Received by the editors June 5, 2000 and, in revised form, September 18, 2000.

1991 Mathematics Subject Classification. Primary 13D40, 13A30, 13 H10.

Key words and phrases. Hilbert-Kunz, multiplicity, regular.

The first author was partially supported by the NSF. 
$e_{H K}(\mathbf{m}, R)$. We write $e_{H K}(I)=e_{H K}(I, R)$. Theorem 1.1 above proves that $e_{H K}(R) \geq 1$, and if $R$ is regular, $e_{H K}(R)=1$. This led to a natural question, probably first posed by Kunz in conversations:

Question 1.3. If $(R, \mathbf{m})$ is a Noetherian unmixed local ring of positive prime characteristic, and $e_{H K}(R)=1$, is $R$ regular?

Recall that a local ring $R$ is unmixed if $\operatorname{dim}(\hat{R})=\operatorname{dim}(\hat{R} / Q)$ for every associated prime $Q$ of $\hat{R}$. The same assumption is needed in the famous result of Samuel (in the case where $R$ contains a field $[\mathrm{Sa}]$ ) and Nagata (in the general case) that the usual multiplicity of an unmixed local Noetherian ring is 1 iff the ring is regular.

Theorem 1.4 ([Na, Theorem 40.6]). Let $R$ be an unmixed Noetherian local ring, not necessarily of characteristic $p>0$. If $R$ has multiplicity one, then $R$ is regular.

In recent beautiful and intricate work, K.-I. Watanabe and K. Yoshida WY] Theorem 1.5] have shown that Question 1.3 has a positive answer. In this paper we give a new proof, which avoids some of the harder parts in the proof of Watanabe and Yoshida; in particular we avoid reference to standard systems of parameters and even to the theory of tight closure, although the present proof was inspired through considerations involving tight closure.

When $R$ is regular, the Hilbert-Kunz multiplicity is easy to compute, due to the exactness of Frobenius. One can easily prove (see [BH, Ex. 8.2.10]):

Proposition 1.5. Let $(R, \mathbf{m})$ be a regular local Noetherian ring of prime characteristic $p>0$ and dimension $d$. Let $I$ be an m-primary ideal. Then for all $q$,

$$
\lambda\left(R / I^{[q]}\right)=q^{d} \cdot \lambda(R / I) .
$$

In particular, $e_{H K}(I)=\lambda(R / I)$ for all $\mathbf{m}$-primary ideals $I$.

\section{Preliminaries}

The next lemma and its corollaries are very useful in studying the length of $R / I^{[q]}$ for an m-primary ideal. They can be found in [WY] Proposition 4.1, Lemma 4.2] in slightly different forms. The filtration argument used in the proof can also be found in [Ha, Proposition 5.2.1]. We include the proof here for completeness.

Lemma 2.1. Let $(R, \mathbf{m})$ be a Noetherian local ring of characteristic $p>0$. Let $I \subseteq J$ be two ideals with $I \mathbf{m}$-primary (we allow $J=R$ ). Then

$$
\lambda\left(R / I^{[q]}\right) \leq \lambda(J / I) \cdot \lambda\left(R / \mathbf{m}^{[q]}\right)+\lambda\left(R / J^{[q]}\right) .
$$

Proof. Set $s=\lambda(J / I)$. Take a filtration of $I \subseteq J \subseteq R$,

$$
I=J_{0} \subsetneq J_{1} \subsetneq J_{2} \subsetneq \cdots \subsetneq J_{s}=J \subseteq R
$$

so that $\lambda\left(J_{i} / J_{i-1}\right)=1$, i.e., $J_{i} / J_{i-1} \cong R / \mathbf{m}, \forall i=1,2, \ldots, s$. That is to say, $J_{i}=\left(J_{i-1}, x_{i}\right)$ for some $x_{i} \in J_{i}$ such that $J_{i-1}: x_{i}=\mathbf{m}$.

For every $q=p^{e}$, there is a corresponding filtration of $I^{[q]} \subseteq J^{[q]} \subseteq R$

$$
I^{[q]}=J_{0}^{[q]} \subseteq J_{1}^{[q]} \subseteq J_{2}^{[q]} \subseteq \cdots \subseteq J_{s}^{[q]}=J^{[q]} \subseteq R,
$$

where $J_{i}^{[q]} / J_{i-1}^{[q]} \cong R /\left(J_{i-1}^{[q]}: x_{i}^{q}\right)$, which is a homomorphic image of $R / m^{[q]}$, for every $i=1,2, \ldots, s$. So $\lambda\left(J_{i}^{[q]} / J_{i-1}^{[q]}\right) \leq \lambda\left(R / \mathbf{m}^{[q]}\right)$. Therefore $\lambda\left(R / I^{[q]}\right) \leq \lambda(J / I)$. $\lambda\left(R / \mathbf{m}^{[q]}\right)+\lambda\left(R / J^{[q]}\right)$. 
Corollary 2.2. Let $(R, \mathbf{m})$ be a Noetherian local ring of characteristic $p>0$. Let $I$ be an $\mathbf{m}$-primary ideal of $R$. Then

(1) $\lambda\left(R / I^{[q]}\right) \leq \lambda(R / I) \cdot \lambda\left(R / \mathbf{m}^{[q]}\right)$.

(2) If $I \subseteq J$, then $e_{H K}(I, R) \leq \lambda(J / I) e_{H K}(R)+e_{H K}(J, R)$.

Proof. In case (1), we take $J=R$ and apply Lemma 2.1. In case (2) the corollary follows from Lemma 2.1 by dividing by $q^{\operatorname{dim} R}$ and then taking the limits.

An important ingredient in our proof is a calculation which shows that, for some ideals, the Hilbert-Kunz multiplicity is well-behaved.

Theorem 2.3. Let $(R, \mathbf{m})$ be a Noetherian local ring of characteristic $p>0$ and dimension $d$. Let $J$ be an ideal such that $\operatorname{dim} R / J=1$ and height $J=d-1$. Assume that $x \in R$ is a non-zerodivisor in $R / J$, and set $I=(J, x)$. Assume that $R_{P}$ is regular for every minimal prime $P$ above $J$. Then

$$
e_{H K}(I, R) \geq \lambda(R / I) .
$$

Proof. Using the properties of the usual multiplicity of parameter ideals, the associativity formula for the usual multiplicity, and (1.5.1), we have

$$
\begin{aligned}
e_{H K}(I, R) & =\lim _{q \rightarrow \infty} \frac{1}{q^{d}} \cdot \lambda\left(R / I^{[q]}\right)=\lim _{q \rightarrow \infty} \frac{1}{q^{d}} \cdot \lambda\left(R /\left(J^{[q]}, x^{q}\right)\right) \\
& \geq \lim _{q \rightarrow \infty} \frac{1}{q^{d}} \cdot e\left(x^{q} ; R / J^{[q]}\right) \\
& =\lim _{q \rightarrow \infty} \frac{q}{q^{d}} \cdot e\left(x ; R / J^{[q]}\right)=\lim _{q \rightarrow \infty} \frac{1}{q^{d-1}} \cdot e\left(x ; R / J^{[q]}\right) \\
& =\lim _{q \rightarrow \infty} \frac{1}{q^{d-1}} \cdot \sum_{P \in \min (R / J)} e(x ; R / P) \cdot \lambda_{R_{P}}\left(R_{P} / J_{P}^{[q]}\right) \\
& =\lim _{q \rightarrow \infty} \frac{1}{q^{d-1}} \cdot \sum_{P \in \min (R / J)} e(x ; R / P) \cdot q^{d-1} \cdot \lambda_{R_{P}}\left(R_{P} / J_{P}\right) \\
& =\lim _{q \rightarrow \infty} \sum_{P \in \min (R / J)} e(x ; R / P) \cdot \lambda_{R_{P}}\left(R_{P} / J_{P}\right) \\
& =\sum_{P \in \min (R / J)} e(x ; R / P) \cdot \lambda_{R_{P}}\left(R_{P} / J_{P}\right)=e(x ; R / J)=\lambda(R /(J, x)) \\
& =\lambda(R / I) .
\end{aligned}
$$

Remark 2.4. One might suspect equality in every case in Theorem 2.3. However the following example provided by the referee shows that inequality is the best one can do: Let $R=k[[x, y, z]] /\left(x y-z^{n}\right), J=(y, z), I=m$. Then $e_{H K}(I)=(2 n-1) / n>$ $\lambda(R / I)=1$.

However, if in addition we assume that $e_{H K}(R)=1$, then it follows from Theorem 2.3 and Corollary 2.2 that in fact

$$
e_{H K}(I, R)=\lambda(R / I) .
$$

However, we will not use this equality in the sequel. In any case, after we prove that $e_{H K}(R)=1$ implies the regularity of $R, e_{H K}(I)=\lambda(R / I)$ for all $m$-primary ideals $I$. 


\section{A CRITERION FOR REGULAR RINGS}

The critical step in proving our main result is in constructing an $\mathbf{m}$-primary ideal $I \subseteq \mathbf{m}^{[p]}$ such that $e_{H K}(I) \geq \lambda(R / I)$. In the paper of Watanabe and Yoshida WY], this step also played an important role. Their construction was done by taking $I$ to be an ideal generated by parameters. However, to prove the inequality it was necessary for them to first prove the ring $R$ is forced to be Cohen-Macaulay if its Hilbert-Kunz multiplicity is one; this is the difficult part of their proof, and required tools from the theory of tight closure and work on standard systems of parameters. We are able to entirely avoid this point by focusing our attention on ideals $I$ which are not necessarily generated by parameters. Here is the theorem of Watanabe and Yoshida [WY] Theorem 1.5].

Theorem 3.1. Let $(R, \mathbf{m})$ be an unmixed Noetherian local ring of prime characteristic $p>0$. If $e_{H K}(R)=1$, then $R$ is regular.

Proof. Since the Hilbert-Kunz multiplicity of $R$ is the same as that of its completion, we may assume $R$ is complete. The associativity formula for Hilbert-Kunz multiplicity shows that

$$
e_{H K}(R)=\sum_{P} e_{H K}(R / P) \cdot \lambda\left(R_{P}\right)
$$

where the sum is over all minimal primes of maximal dimension. Since $e_{H K}(R)=1$, we deduce that $R$ can have only one minimal prime $P$ and $R_{P}$ has to be field, i.e., $P_{P}=0$. Hence $P=0$ since $R \backslash P$ consists of non-zerodivisors. Thus $R$ is a domain.

It suffices to prove that $\lambda\left(R / \mathbf{m}^{[p]}\right) \leq p^{d}$ (where $d=\operatorname{dim}(R)$ ) as then Theorem 1.1 (1) first gives equality, and then Theorem 1.1 (2) gives that $R$ must be regular.

The singular locus of $R$ is closed and not equal to $\operatorname{Spec}(R)$. It follows we can choose a prime $P$ such that $\operatorname{dim}(R / P)=1$ and $R_{P}$ is regular. (If not, the intersection of all primes $Q$ such that $\operatorname{dim}(R / Q)=1$ must be nonzero. Choose $f \neq 0$ in this intersection and extend $f$ to a full system of parameters $g_{1}, \ldots, g_{d-1}, f$. Taking a minimal prime $Q$ over $\left(g_{1}, \ldots, g_{d-1}\right)$ gives a contradiction.) Since the intersection of the symbolic powers of $P$ is zero and $R$ is complete, Chevalley's lemma gives that some sufficiently large symbolic power of $P$ lies inside $\mathbf{m}^{[p]}$. Call this symbolic power $J$. Choose $x \in \mathbf{m}^{[p]}$ such that $x \notin P$. The ideal $I=(J, x)$ lies in $\mathbf{m}^{[p]}$ and satisfies the hypothesis of Theorem 2.3. Hence

$$
e_{H K}(I) \geq \lambda(R / I)
$$

On the other hand we have $e_{H K}(I, R) \leq \lambda\left(\mathbf{m}^{[p]} / I\right) \cdot e_{H K}(R)+e_{H K}\left(\mathbf{m}^{[p]}, R\right)=$ $\lambda\left(\mathbf{m}^{[p]} / I\right)+e_{H K}\left(\mathbf{m}^{[p]}, R\right) \leq \lambda\left(\mathbf{m}^{[p]} / I\right)+\lambda\left(R / \mathbf{m}^{[p]}\right)$, by Lemma 2.1 and Corollary 2.2 .

That is to say,

$$
\begin{aligned}
\lambda\left(\mathbf{m}^{[p]} / I\right)+\lambda\left(R / \mathbf{m}^{[p]}\right) & =\lambda(R / I) \leq e_{H K}(I, R) \\
& \leq \lambda\left(\mathbf{m}^{[p]} / I\right)+e_{H K}\left(\mathbf{m}^{[p]}, R\right) \\
& \leq \lambda\left(\mathbf{m}^{[p]} / I\right)+\lambda\left(R / \mathbf{m}^{[p]}\right),
\end{aligned}
$$

which forces $\lambda\left(R / \mathbf{m}^{[p]}\right)=e_{H K}\left(\mathbf{m}^{[p]}, R\right)$. However,

$$
e_{H K}\left(\mathbf{m}^{[p]}, R\right)=\underline{\lim _{\longrightarrow}} \frac{\lambda\left(R / \mathbf{m}^{[p q]}\right)}{q^{d}}=\underline{\lim _{\longrightarrow}} \frac{p^{d} \cdot \lambda\left(R / \mathbf{m}^{[p q]}\right)}{(p q)^{d}}=p^{d} \cdot e_{H K}(R)=p^{d} .
$$


Together the equalities imply that $\lambda\left(R / \mathbf{m}^{[p]}\right)=p^{d}$, which implies that $R$ is regular by Theorem 1.1.

Remark 3.2. An alternate proof could be given which by induction allows one to assume that $R_{Q}$ is regular for all primes $Q \neq \mathbf{m}$. This is due to the upper semicontinuity of the Hilbert-Kunz multiplicity. This was shown by Kunz Ku2. We append a shorter proof, much in the spirit of our simplification of the main theorem.

Theorem 3.3 ( $\mathrm{Ku} 2$ Corollary 3.8]). Let $(R, \mathbf{m})$ be a Noetherian local ring of prime characteristic $p>0$, and let $P$ be a prime ideal of $R$ such that height $(P)+$ $\operatorname{dim}(R / P)=\operatorname{dim}(R)$. Then $e_{H K}\left(R_{P}\right) \leq e_{H K}(R)$. In fact, if $t=\operatorname{dim}(R / P)$, then $q^{t} \cdot \lambda_{R_{P}}\left(\left(R / P^{[q]}\right)_{P}\right) \leq \lambda\left(R / \mathbf{m}^{[q]}\right)$ for every $q=p^{e}$.

Proof. By induction, it is enough to prove the case where $h t(P)=\operatorname{dim}(R)-1$. Notice it suffices to prove the second inequality.

Choose $f \in \mathbf{m}-P$. Then, using the properties of the usual multiplicity of parameter ideals, the associativity formula for the usual multiplicity, we have, for all $q=p^{e}$

$$
\begin{aligned}
\lambda\left(R /(P, f)^{[q]}\right) & =\lambda\left(R /\left(P^{[q]}, f^{q}\right)\right) \\
& \geq e\left(f^{q} ; R / P^{[q]}\right) \\
& =\lambda_{R_{P}}\left(\left(R / P^{[q]}\right)_{P}\right) \cdot e\left(f^{q} ; R / P\right) \\
& =\lambda_{R_{P}}\left(\left(R / P^{[q]}\right)_{P}\right) \cdot q \cdot \lambda(R /(f, P)) .
\end{aligned}
$$

Also, by Corollary 2.2, we know that $\lambda(R /(f, P)) \cdot \lambda\left(R / \mathbf{m}^{[q]}\right) \geq \lambda\left(R /(P, f)^{[q]}\right)$. Hence $\lambda\left(R / \mathbf{m}^{[q]}\right) \geq q \cdot \lambda_{R_{P}}\left(\left(R / P^{[q]}\right)_{P}\right)$ for every $q=p^{e}$.

\section{REFERENCES}

[BH] W. Bruns and J. Herzog, Cohen-Macaulay Rings, vol. 39, Cambridge studies in advanced mathematics, 1993. MR 95h:13020

[Ha] D. Hanes, Special conditions on maximal Cohen-Macaulay modules, and applications to the theory of multiplicities, Thesis, University of Michigan, 1999.

[Ku1] E. Kunz, On Noetherian rings of characteristic p, Amer. J. Math. 98 (1976), 999-1013. MR 55:5612

[Ku2] E. Kunz, Characterizations of regular local rings of characteristic p, Amer. J. Math. 91 (1969), 772-784. MR 40:5609

[Mo] P. Monsky, The Hilbert-Kunz function, Math. Annalen 263 (1983), 43-49. MR 84k:13012

[Na] M. Nagata, Local Rings, Robert E. Kreiger Publishing Co., 1975. MR 57:301

[Sa] P. Samuel, La notion de multiplicité en algébre et en géométrie algèbrique, I \& II, J. Math. Pures. Appl. 30 (1951), 159-274. MR 13:980c MR 13:980d

[WY] K. Watanabe, K. Yoshida, Hilbert-Kunz multiplicity and an inequality between multiplicity and colength, J. Algebra 230 (2000), 295-317. CMP 2000:16

Department of Mathematics, University of Kansas, Lawrence, Kansas 66045

E-mail address: huneke@math.ukans.edu

Department of Mathematics, University of Kansas, Lawrence, Kansas 66045

E-mail address: yyao@math.ukans.edu 